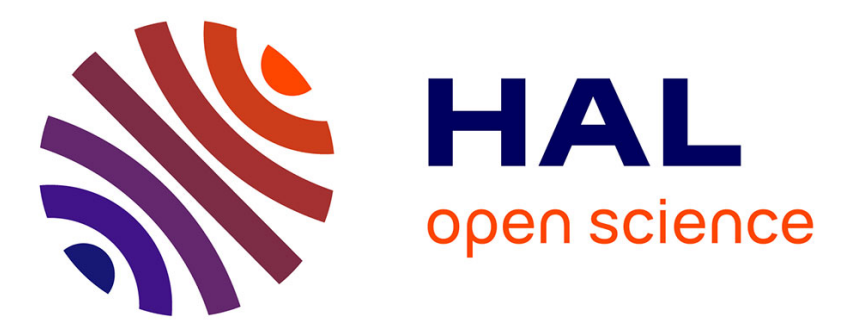

\title{
On the study of mystical materials identified by indentation on power law and Voce hardening solids
}

L. Meng, P. Breitkopf, Balaji Raghavan, Gerard Mauvoisin, Olivier Bartier, Xavier Hernot

\section{- To cite this version:}

L. Meng, P. Breitkopf, Balaji Raghavan, Gerard Mauvoisin, Olivier Bartier, et al.. On the study of mystical materials identified by indentation on power law and Voce hardening solids. International Journal of Material Forming, 2019, 12 (4), pp.587-602. 10.1007/s12289-018-1436-1 . hal-01884269

\section{HAL Id: hal-01884269 \\ https://hal.science/hal-01884269}

Submitted on 18 Oct 2018

HAL is a multi-disciplinary open access archive for the deposit and dissemination of scientific research documents, whether they are published or not. The documents may come from teaching and research institutions in France or abroad, or from public or private research centers.
L'archive ouverte pluridisciplinaire HAL, est destinée au dépôt et à la diffusion de documents scientifiques de niveau recherche, publiés ou non, émanant des établissements d'enseignement et de recherche français ou étrangers, des laboratoires publics ou privés. 


\title{
On the study of mystical materials identified by indentation on power law and Voce hardening solids
}

\author{
L. Meng ${ }^{a, b}$ • P. Breitkopf $f^{b}$. \\ B. Raghavan ${ }^{c}$. G. Mauvoisin ${ }^{d}$. O. Bartier ${ }^{d}$. X. Hernot Her $^{d}$ \\ L.Meng (E-mail: liang.meng@nwpu.edu.cn) \\ ${ }^{a}$ State IJR Center of Aerospace Design and Additive Manufacutring, Northwestern Poly- \\ technical University, Xi'an, Chine \\ ${ }^{b}$ Sorbonne Universités, Université de Technologie de Compiègne, CNRS, Laboratoire Rober- \\ val, UMR 7337, Centre de Recherches de Royallieu, CS 60319, 60200, Compiègne Cedex, \\ France

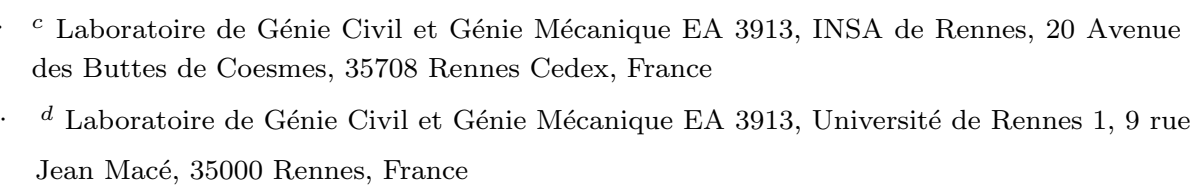

\begin{abstract}
We conduct an incisive investigation of the existence of a one-toone correspondence between a material's elastoplastic properties and its indentation responses, with particular emphasis on the residual imprint. We first unravel a so-called "mystical material" pair reported by Chen et al. (2007) by examining the specimens' post-indentation morphologies, despite using a single self-similar indenter. Next, using Metric Multidimensional Scaling (MDS), we mitigate the mystical material issue for materials hardening according to the popular Hollomon's power law equation. In contrast, the same exact protocol reveals the absence of a one-to-one correspondence between the single indentation response and Voce hardening parameters. To alleviate this, we propose a multi-depth indentation strategy.
\end{abstract}

Keywords Plasticity · strain hardening · characterization · mystical materials · indentation imprint · Multidimensional Scaling

\section{Introduction}

The instrumented indentation test has grown out of its humble origins as little more than a surface hardness testing procedure [1] and slowly but surely established itself as a viable non-destructive alternative to the conventional tensile test under certain conditions. Today it is widely used to probe the 
constitutive relationships of a variety of engineering materials [2-6]. However, the available literature on indentation-based mechanical characterization indicates that the uniqueness, or lack thereof, of the solution to the final inverse identification problem has, particularly in recent years, become a focal point of research [7-13], calling into question the quality of the solution(s) obtained. According to [11], the difficulty in studying the non-uniqueness issue is partly due to the absence of an explicit relationship between material's properties and its complex indentation responses.

Thus far, the non-uniqueness issue has been established mainly in the context of the loading-unloading $(P-h)$ curve, and it is now widely recognized that this problem is particularly severe when self-similar indenters (mainly conical ones) are used for the identification of Hollomon's hardening parameters $[14,15]$. Using dimensional analysis, Chen et al. introduced a series of explicit formulations to determine the so-called "mystical material" pairs with distinct elastoplastic properties yet almost indistinguishable $P$ - $h$ curves [11]. To tackle this problem, he proposed two alternative techniques (dual-sharp and spherical indentation) to yield a unique material parameter set $[16,17]$.

Spurred on by the development of high-resolution scanning probe microscopy techniques (e.g., Atomic Force Microscope (AFM)), other solutions were also provided in [18-22], where the residual imprint, measured with high precision and accuracy, were taken into account. For example, since 2007, [23] has combined the $P$ - $h$ curve with the imprint to probe the constitutive parameters of thin layers on substrate. The indenter was forced into the specimen until a maximum penetration depth of only $0.4 \mu \mathrm{m}$, and the residual imprint was mapped by means of AFM. Later, [24] adopted both a profilometer and a portable microscope to measure the residual imprint on a metal specimen being characterized. The accuracy of these measurements was also compared with the sensing device of the indentation tool, showing an acceptable difference of $2 \mu \mathrm{m}$. Another example of the use of imprint in nano-scale is found in [21], where the residual profile on human bone was also measured using AFM.

It is glaringly obvious that the surface measuring techniques available today allow for a greater perspective on material characterization on account of the larger amount of exploitable data available. While taking the imprint into account would ostensibly render the identification procedure more cumbersome, both experimentally as well as in terms of convergence of the algorithm, it would provide us with an opportunity to take a deeper look at the nonuniqueness issue in the inverse identification problem.

The last few decades have seen a steady rise in the number and complexity of applications of machine learning/data mining techniques in the field of mechanics [25-31] for two reasons: first, the modern-day maturity of computational engineering in simulating complex phenomena with attention to details; and second, the development of modern techniques trending towards high-precision measurements on a smaller and smaller scale, both producing huge amounts of data. In this regard, dimensionality reduction algorithms, allowing physical data to be condensed in a very light and efficient manner, 
can be quite appealing to "wisely" study the material plasticity encoded in the indentation responses, as is performed in the current work.

In this paper, we conduct a thorough investigation of the identifiability issue for materials hardening according to either the Hollomon's power law equation [22] or the less frequently used Voce law [32], within a condensed space of indentation responses. The sufficient conditions for uniquely identifying the two constitutive laws from the measured indentation responses are carefully examined. Using Metric Multidimensional Scaling (MDS), we explore the material parameter space for the possible existence of non-unique solutions to the inverse problem. The insights gleaned from this reduced-order space are validated by the identification procedure using a non-linear manifold learning algorithm [33-35].

The remainder of the paper is organized in the following manner: we first present the simulation FE model and the fundamental hypothesis in Sect.2; Sect. 3 focuses on targeting and adapting MDS to the indentation framework; we then illustrate in Sect. 4 the existence of a unique solution to the identification problem assuming power law hardening; the identification of Voce hardening parameters and the difficulties encountered are studied in Sect.5 and 6 , respectively; finally, Sect.7 introduces a multi-depth indentation technique for the unique identification of the Voce parameters.

\section{Overall concepts and problem description}

In a typical inverse identification procedure, one expects that there exists a one-to-one correspondence between the recorded data (response) and the desired material parameters defining a postulated constitutive law. Unfortunately, the indentation problem is frequently ill-posed, and the existence of different "sibling" materials with distinct properties but almost identical indentation curve has been revealed by more than one author $[36,10]$. Representative work has been reported in [11], where the authors employed the term "mystical materials" to interpret this phenomenon.

\section{1 "Mystical" material pairs}

In [11], an example mystical material pair $\left(\mathrm{M}_{1}, \mathrm{M}_{2}\right)_{\theta=74^{\circ}}$ was obtained by enforcing the equality of the curvature of the loading curve using a conical indenter with apex angle $\theta=74^{\circ}$. The $P$ - $h$ curves for the two materials are observed to overlap with each other, Fig.1 (a). Their uniaxial $\sigma-\varepsilon$ curves are given in the top-left inset of Fig.1 (a). As pointed out by Chen et al., the curves may be distinguished by using dual-sharp indenters, and the difference between two obtained $P$ - $h$ curves increases for extreme angles, e.g., $\theta=63.14^{\circ}$ and $\theta=75.79^{\circ}$ due to differences between the plastic strain fields underneath differently shaped indenter tips [17]. On the other hand, as pointed out in [11], any apex angle can lead to an infinite number of "mystical material" pairs, 
whose $\sigma-\varepsilon$ curves are observed to intersect with each other at a "representative strain" [15].

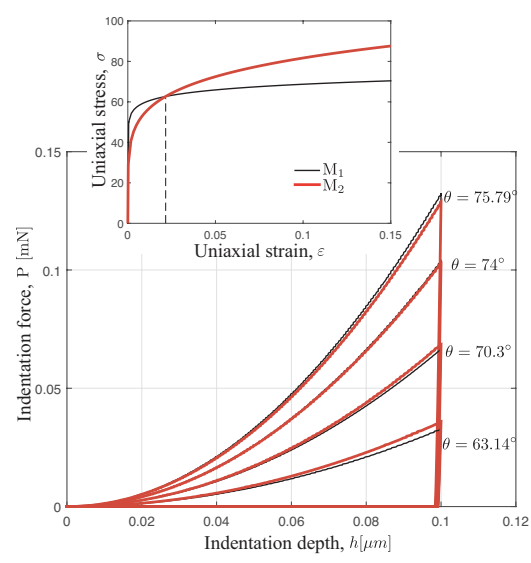

(a)

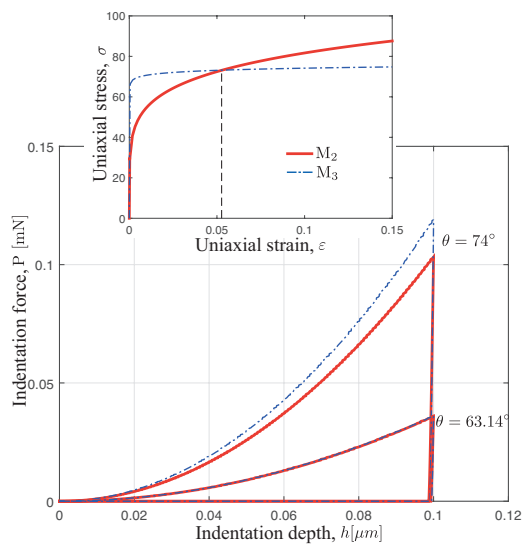

(b)

Fig. 1: Two mystical material pairs (Hollomon's law): (a) $\left(\mathrm{M}_{1}, \mathrm{M}_{2}\right)_{\theta=74^{\circ}}[11]$ and (b)

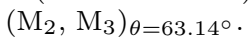

For the purpose of comparison, we also locate a pair of materials that can be readily distinguished using a conical indenter of $74^{\circ}$, but not another angle, say $63.14^{\circ}$. Let us reconsider $\mathrm{M}_{2}$ as the reference material, and $\mathrm{M}_{3}$ is one of its mystical siblings w.r.t. a conical indenter of apex angle $\theta=63.14^{\circ}$. Note that $\mathrm{M}_{3}$ is obtained by inverse identification [34] instead of using the empirical formulas provided by [11]. Here, the simulated indentation response ( $P$ - $h$ curve) of $\mathrm{M}_{2}$ has been considered as the target shape, and an initial point was chosen far away from $\mathrm{M}_{1}$. From Fig.1 (b), we see that, with $\theta=74^{\circ}, \mathrm{M}_{2}$ and $\mathrm{M}_{3}$ can be readily distinguished, but this is not the case for $\theta=63.14^{\circ}$. Tab.1 compares both mystical pairs w.r.t. the two cones. Note also that other alternatives do exist, and may be obtained by convergence from different initial points.

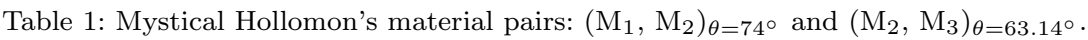

\begin{tabular}{cccc}
\hline Material & $E(\mathrm{GPa})$ & $\sigma_{y}(\mathrm{MPa})$ & $n$ \\
\hline $\mathrm{M}_{1}$ & 100 & 50 & 0.06 \\
$\mathrm{M}_{2}$ & 110 & 29.34 & 0.173 \\
$\mathrm{M}_{3}$ & 150 & 66.26 & 0.021 \\
\hline
\end{tabular}

2.2 Simulation hypothesis 
In concordance with $[37,11]$, we develop a numerical model to further investigate the mystical material pairs, based on the following assumptions:

- The compliance of the measuring system and of the indenter tip are negligible;

- The bulk materials are assumed to be homogeneous and are free from residual stresses;

- The penetration depth of the indenter tip is sufficient to neglect size and tip bluntness effects;

- Young's modulus and Poisson's ratio are assumed to be known a priori.

While some of these hypothesis may be too restrictive for application to certain cases, e.g., thin-film materials [7,38-40,23], they are somewhat acceptable for studying the non-uniqueness issue encountered during inverse identification of bulk materials. We concern ourselves in this work with isotropic materials with rate-independent associated $J_{2}$ plasticity, i.e., nearly incompressible and isotropic strain hardening; the rheological behavior is typically given by expressing flow stress as either a function of total strain (e.g., Hollomon's law) or as a piecewise continuous function with an elastic and plastic components and strain hardening parameters (e.g., Voce law).

\subsection{Hardening models}

In this subsection, we briefly recall two different isotropic hardening models with the aim of establishing the parameters to be identified.

\subsubsection{Hollomon's equation (power law)}

Due to its simplicity, the power law is frequently employed to describe the isotropic hardening behavior of metals and alloys [41]. By enforcing continuity at the elastic limit, the relationship between equivalent stress $(\sigma)$ and total strain $(\varepsilon)$ may be given as

$$
\sigma=\sigma_{y}\left(\frac{E}{\sigma_{y}}\right)^{n} \varepsilon^{n} .
$$

The two hardening parameters, the initial elastic limit $\sigma_{y}$ and the hardening coefficient $n$, are to be identified, while Young's modulus $E$ is generally known a priori.

\subsubsection{Three-parameter Voce equation}

Many important engineering materials deviate significantly from simple power law hardening $[42,32,43]$. For the aluminum alloy EN AW-5754F characterized using the Hollomon's equation in [13], the authors observed a pronounced difference between the stress-strain curve measured from the tensile test and 
that plotted using the identified parameter values. The Voce law $[44,45]$, exhibiting a saturation stress $\left(\sigma_{s}\right)$ at high strain level, is likely suitable for this family of alloys. Unlike Eq.(1), the Voce uniaxial $\sigma-\varepsilon$ relationship is defined in piecewise fashion [32]

$$
\left\{\begin{array}{l}
\sigma=E \varepsilon ; \varepsilon<\sigma_{y} / E \\
\sigma=\sigma_{y}+Q\left(1-e^{-\gamma \varepsilon_{p}}\right)
\end{array}\right.
$$

in which $Q=\sigma_{s}-\sigma_{y}$ is the difference between the saturation stress $\sigma_{s}$ and the initial elastic limit. The parameter $\gamma$ controls how "rapidly" the stress approaches the saturation level, and it may vary over a wide range, capable of accommodating large class of strain hardening engineering materials: from bilinear elastoplastic $(\gamma=2)$ to elastic-perfectly plastic $(\gamma=300)$, Fig.2.

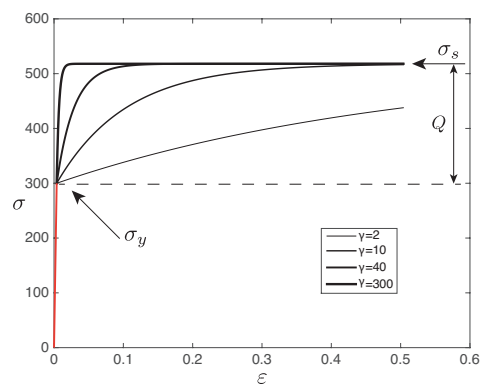

Fig. 2: Voce law with three hardening parameters: $\sigma_{y}, Q$ and $\gamma$.

\subsection{Forward finite element model}

Assuming isotropic materials, we adopt the axisymmetric model as in [34], a $50 \mathrm{~mm} \times 50 \mathrm{~mm}$ specimen, large enough to approximate a semi-infinite halfspace. The FE mesh, relatively dense in the central contact area, progressively coarsens outwards. The element size around the indenter tip is as small as $3 \mu \mathrm{m}$. It should be noticed that model error shall present itself inevitably in view of the assumptions made in Sect. 2.2. For this reason, the difference between simulated indentation responses and physical trials are difficulty to be quantified with precision. However, the convergence of the model has been checked and the indentation system modeled with ABAQUS/Standard converges without further remeshing.

Berkovich and Vickers indenters, though popular in engineering practice, are replaced with a conical indenter with an included apex angle $\theta=70.3^{\circ}$, in view of the equivalent projection area/depth ratio [11]. Other apex angles are also studied. We consider on the other hand a spherical indenter with $R=0.5 \mathrm{~mm}$ since it presents the advantage of an additional length-scale when compared to self-similar indenters [46]. The conical indenters, usually made of diamond, are considered as rigid bodies, while the spherical indenter tip, 
made of Tungsten Carbide, is modeled with $E=600 \mathrm{Gpa}$ and $v=0.23$ (elastic properties measured by ultrasound). Depth-controlled FE simulations, giving a slightly better-conditioned problem in comparison with load-controlled ones [13], are preferentially adopted.

For the contact between the indenter and the specimen, we assume Coulomb friction. Our literature review reveals that the friction effect is seldom considered: some assign a pre-defined friction coefficient, while others simply assume frictionless contact. We must be aware that this choice could significantly affect the imprint mapping.

We perform simulations to obtain a series of indentation imprints for the mystical pair $\left(\mathrm{M}_{1}, \mathrm{M}_{2}\right)_{\theta=74^{\circ}}$, varying the friction coefficient from 0 to 0.5 . The pile-up heights are plotted against diverse maximum penetration depths in Fig.3. We notice that less friction $(\mu \leq 0.2)$ or frictionless contact lead to higher pile-up heights which may be explained by sliding contact behavior. Moreover, the pronounced difference between pile-up heights w.r.t. different materials suggests that a coefficient inferior to 0.2 can be favorable for the distinction of mystical pair. In practice, such small friction coefficient can be well obtained by polishing the specimen before indentation test. For example, the three specimens investigated in [22] were polished with a fine emery paper (up to 1200 grit) and diamond suspensions $(6$ and $3 \mu \mathrm{m}$ ) for the purpose of avoiding roughness-related uncertainties. By combining with empirical experiences, a friction coefficient of 0.1 was adopted, and the characterization results from imprint mapping are quite acceptable in comparing with the $\sigma$ - $\varepsilon$ curve obtained from tensile test.

That said, we must address the use of imprint is based on the premise that the friction is well-characterized, which we think is practically possible. The influence of the choice of friction coefficient on the reliability of material characterization, though beyond the scope of current research, deserves further examination. In the following, a friction coefficient of 0.15 is adopted, following $[47,11]$.

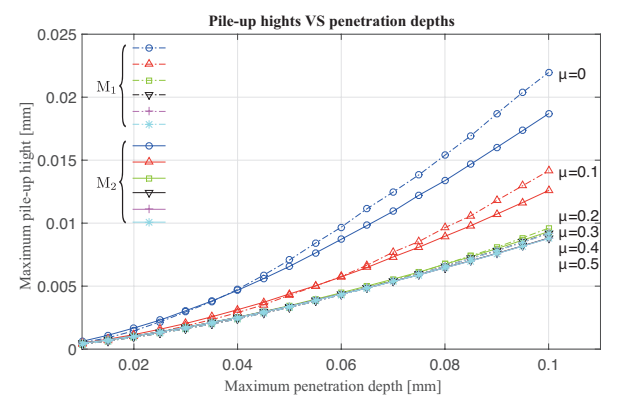

Fig. 3: The influence of friction coefficient on the maximum pile-up observed on imprints. 


\section{Dimensionality reduction approach (MDS) applied to material characterization}

3.1 Material calibration in reduced shape space

The aim of inverse identification is to minimize the discrepancy between the experimental and the simulated responses, thus requiring the computation of distances between high-dimensional data sets. In the context of indentation, these data sets could either be the residual imprint containing the vertical displacements of nodes along the imprint profile [13], or the pointwise forcedisplacement curves [48]. Both of them may be considered as "special shapes" existing in a high-dimensional "shape space". With the aim of providing an easy-to-interpret description [49], we build a low-dimensional embedding based on MDS, preserving pairwise distances between indentation instances in both the original as well as the embedded spaces.

Let $\boldsymbol{s}^{(i)}=\mathbf{s}\left(\boldsymbol{\mu}_{i}\right) \in \mathbb{R}^{N}, i=1, \cdots, M$ denote a series of high-dimensional data sets for different materials with varying parameter values $\boldsymbol{\mu}$. Without loss of generality, we may assume that the observations are centered

$$
\frac{1}{M} \sum_{i=1}^{M} \boldsymbol{s}^{(i)}=\mathbf{0} \in \mathbb{R}^{N} .
$$

The matrix of squared pairwise distances $\boldsymbol{D}$ between the input patterns

$$
D_{i j}=\left\|\boldsymbol{s}^{(i)}-\boldsymbol{s}^{(j)}\right\|^{2}, \quad i, j=1, \cdots, M
$$

leads by transformation to the Gram matrix

$$
\boldsymbol{G}=\frac{1}{2}\left(\boldsymbol{I}_{N}-\boldsymbol{u} \boldsymbol{u}^{\mathrm{T}}\right) \boldsymbol{D}\left(\boldsymbol{I}_{N}-\boldsymbol{u} \boldsymbol{u}^{\mathrm{T}}\right)
$$

where $\boldsymbol{I}_{N}$ is the $N \times N$ identity matrix and $\boldsymbol{u}=\frac{1}{\sqrt{N}}(1, \cdots, 1)^{\mathrm{T}}$.

The corresponding coordinates $\boldsymbol{\alpha}^{(i)}$ of the inputs in the embedded reducedorder space $\mathbb{R}^{d}(d<<N)$ minimize

$$
J(\boldsymbol{\alpha})=\sum_{i j}\left(\boldsymbol{s}^{(i)} \boldsymbol{s}^{(j)}-\boldsymbol{\alpha}^{(i)} \boldsymbol{\alpha}^{(j)}\right)^{2}, \quad i, j=1, \cdots, M,
$$

and are obtained by the spectral decomposition of $\boldsymbol{G}$, which may also be presented by inner products

$$
G_{i j}=\boldsymbol{s}^{(i)} \boldsymbol{s}^{(j)}, \quad i, j=1, \cdots, M .
$$

Denoting the first $m$ eigenvectors by $\boldsymbol{v}_{j}$ and the corresponding eigenvalues by $\lambda_{j}$, we obtain:

$$
\boldsymbol{\alpha}_{j}=\sqrt{\lambda_{j}} \boldsymbol{v}_{j}, \quad j=1, \cdots, m .
$$

Although based on different geometric intuitions, MDS yields the same output as the common Principal Component Analysis (PCA), since $\boldsymbol{G}=\boldsymbol{S}^{\mathrm{T}} \boldsymbol{S}$ has the 
same rank and the same eigenvalues as the covariance matrix $\boldsymbol{C}=\frac{1}{M} \boldsymbol{S} \boldsymbol{S}^{\mathrm{T}}$ with $\boldsymbol{S}=\left[\boldsymbol{s}^{(1)}, \boldsymbol{s}^{(2)}, \cdots, \boldsymbol{s}^{(M)}\right]$. For these reasons, a PCA-based algorithm [34,26, $50,13]$ may be used in the MDS reduced-dimensional space along with the identification error

$$
\epsilon(\boldsymbol{\mu})=\left\|\boldsymbol{s}^{\exp }-\boldsymbol{s}(\boldsymbol{\mu})\right\|_{L_{2}}=\left\|\boldsymbol{\alpha}^{\exp }-\boldsymbol{\alpha}(\boldsymbol{\mu})\right\|_{L_{2}}
$$

where $\boldsymbol{s}^{\exp }$ refers to the experimental measurement, and $\boldsymbol{\alpha}^{\exp }$ its projection in the reduced shape space. Following [51], we quantify the level of agreement between the experimental and simulated indentation responses with a dimensionless indicator: "goodness of fit"

$$
g=1-\frac{\epsilon}{\left\|s^{*}\right\|_{L_{2}}},
$$

with a value of 1 representing a "perfect fit".

\subsection{An illustrative example of MDS application}

To illustrate the application of MDS in reducing the dimensionality of physical data, we consider first the uniaxial stress strain $(\sigma-\varepsilon)$ curves of Hooke's law. A group of 50 tensile curves are sampled varying $E \in[200 \mathrm{GPa}, 800 \mathrm{GPa}]$, and they are directly considered as high-dimensional MDS input "shapes", each characterized by a vector of stresses interpolated on a fixed grid of strains varying between 0 and 1. Fig.4 (a) shows a series of representative stress-strain curves, while a reduced space into which the synthetic curves are projected is observed in (b). According to Eq.(3-8), each $\sigma$ - $\varepsilon$ curve is now represented by a single point in $\alpha$-space. The set of stress-strain behaviors of perfectly elastic materials is readily observed to lie on a straight line in the reduced space.

On the other hand, another reduced shape is constructed w.r.t. the $P$ - $h$ curves (Fig.4 (c)) of the sampled elastic materials. An aligned distribution of corresponding points in reduced space is observed in Fig.4 (d), with progressively varying density. By examining the elastic properties, we find that the materials (represented by their modulus) located in the dense zone correspond to materials with higher $E$. That said, the one-to-one correspondence between parameter value $E$ and the indentation curve is still valid, leading potentially to a unique solution in indentation-based inverse identification.

\section{On the existence of a unique solution for Hollomon's power law parameters}

4.1 Unraveling mystical materials for Hollomon's hardening law

In $[13,22]$, we have presented a novel image-correlation protocol for identifying plastic power law parameters based solely on using the residual imprint, 

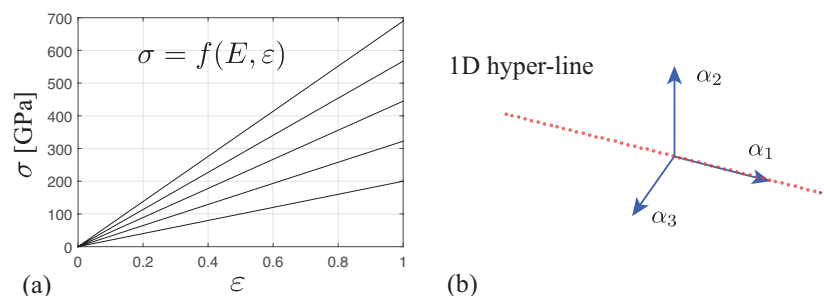

(a)

(b)
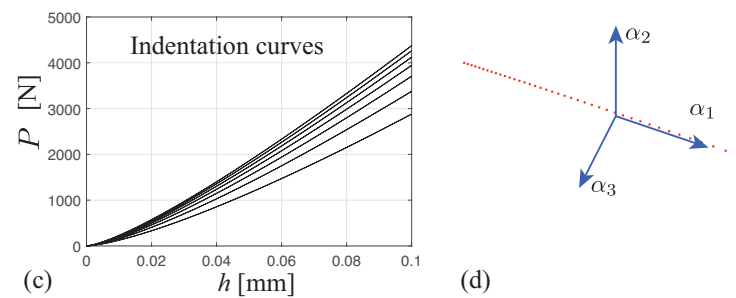

(d)

Fig. 4: An illustrative example of MDS applied to Hooke's law: (a)-(c) Stress-strain curves; and (b)-(d) indentation curves for sampled elastic materials.

and the results showed that the pile-up zone plays a vital role in distinguishing between materials following the Hollomon's hardening law and the nonuniqueness issue appears to be alleviated, at least for spherical indentation. In this section, we attempt to confirm the validity of this observation for the more popular conical indenters.

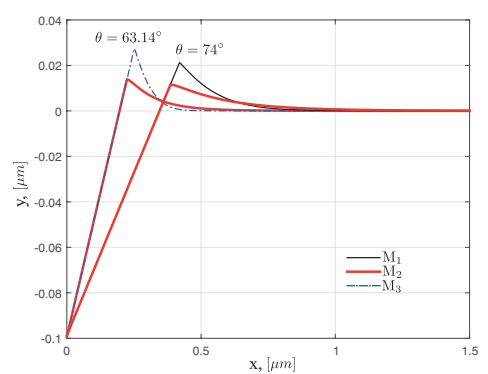

Fig. 5: Distinguishable residual imprints for the two mystical material pairs using conical indenters.

Fig. 5 shows the residual imprints of the two mystical material pairs, $\left(\mathrm{M}_{1}\right.$,

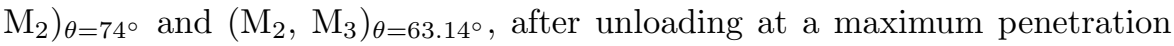
depth $h_{\max }=0.1 \mathrm{~mm}$. Similar to the case of spherical indentation, clear differences are as expected observed around the pile-up zones, and both material pairs are no longer "mystical" when we consider the residual imprint profiles. Therefore, we speculate that the imprint mapping may possibly contain more "complete" information of the material's hardening behavior, thus rendering 
the inverse problem "better-posed", at least as far as isotropic elastoplastic materials with isotropic hardening and rate-independent $J_{2}$ plasticity are concerned, since that corresponds to the constitutive model used for creating the space.

However, we must still be cautious to rule out the existence of mystical materials for the imprint-based inverse problem. In other words, we need to answer the question of whether or not there exist different materials that reveal indistinguishable residual imprints.

\section{2 "Demystifying" materials within the reduced imprint space}

We perform a series of FE simulations over a factorial Design of Experiments (DoE) with $M=11 \times 11=121$ instances and material intervals: $n \in[0.2,0.3]$ and $\sigma_{y} \in[200 \mathrm{MPa}, 240 \mathrm{MPa}]$, assuming a conical indenter $\theta=74^{\circ}$. These parameter intervals have been chosen taking reference to the material properties of C100 steel and (AA2017) aluminum alloy reported previously [34]. Though these intervals may not be large enough to include most metallic materials, they still allow us to depict a small patch of the manifold which is sufficient to investigate the non-uniqueness issue.

Following Eqs.(5-8), every imprint is projected to a single point in the shape space. Since the intrinsic dimensionality of the shape space can not exceed the number of material parameters being manipulated in the DoE $(d=2$ for Hollomon's hardening materials), the reduced indentation responses may be readily visualized in a 3D Cartesian coordinate system $(\mathrm{m}=3)$, Fig.6.

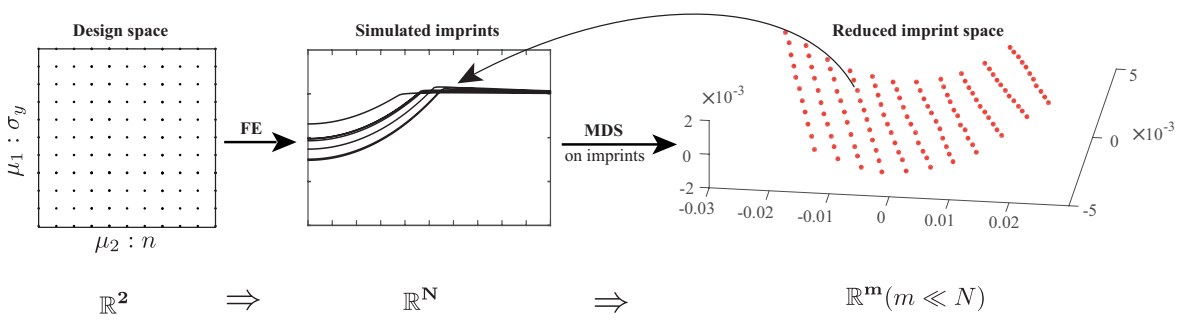

Fig. 6: Correspondence between the DoE and residual imprint mappings in "imprint space".

We observe in the imprint space that all imprints are clearly separated and show a regular distribution (without overlapping or intersection). This clearly illustrates a one-to-one correspondence between the material parameters and the residual imprint mappings, and further suggests the absence of two different material parameter sets that may lead to the same imprint profile upon indenter withdrawal. For this reason, we may consider the inverse identification problem based on the indentation imprint as well-posed, and we 
can successfully demystify the 'mystical siblings' of Hollomon's law by using the residual imprint, even with a single self-similar indenter.

\section{The non-unique identification of Voce parameters based on a single imprint}

We have until now shown that we are capable of uniquely identifying the Hollomon's parameters from a single residual imprint, regardless of the indenter geometry. In the current section, we intend to verify the validity of this conclusion on Voce law. The corresponding $P$ - $h$ curves will be compared only at convergence and purely for the purpose of verification.

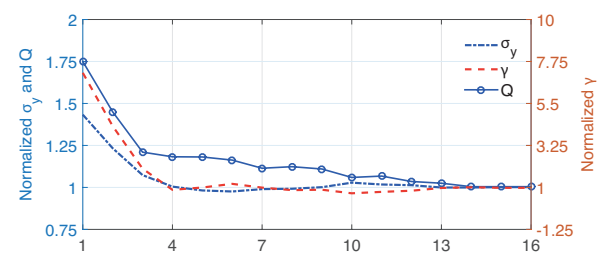

Fig. 7: Convergence summary for the three Voce parameters $\left(\mathrm{M}_{4}\right)$ normalized by their corresponding reference values (initial point $\boldsymbol{\mu}_{A}$ ).

A synthetic imprint is employed as the target shape, and this "pseudoexperimental" response is simulated from the model in Sect.2.4, with a maximal penetration depth $h_{\max }=0.1 \mathrm{~mm}$, and using: $\boldsymbol{\mu}^{*}:\left(\sigma_{y}^{*}, Q^{*}, \gamma^{*}\right)=(300 \mathrm{MPa}, 200 \mathrm{MPa}, 14)$. By using the "Floating search" algorithm $[35,34]$ along with $\beta=0.8$, we attempt to identify the three constitutive parameters.

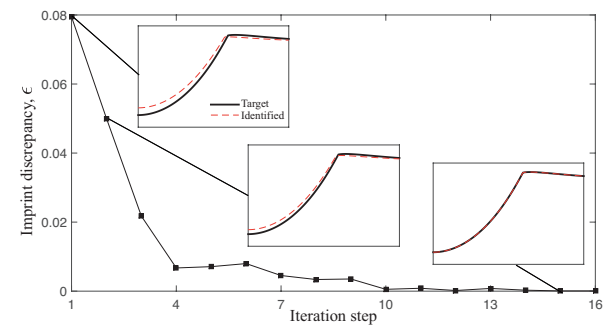

Fig. 8: Minimization of the discrepancy between the simulated $\left(\mathrm{M}_{4}\right)$ and target (pseudo-experimental) imprints (initial point $\boldsymbol{\mu}_{A}$ )

An arbitrarily chosen combination of constitutive parameters $\boldsymbol{\mu}_{A}$ : (430 MPa, $\left.350 \mathrm{MPa}, 100\right)$ is set as the initial guess for iteration. In Fig.7, we present the convergence histories for the three Voce parameters, normalized by their corresponding reference/nominal values, $\sigma_{y}^{*}, Q^{*}$ and $\gamma^{*}$. The identified material is noted as $\mathrm{M}_{4}$, 
and the parameter values are compared with their reference values in Tab. 2 . We observe that by beginning the search from $\boldsymbol{\mu}_{A}$, we are able to retrieve the three parameters with reasonable accuracy at the end of 16 iterations, with a maximum error of $2.14 \%$ being observed for the third parameter $\gamma$. Fig. 8 also shows the iteration history for the cost function Eq.(9). Its steady decline confirms the robustness of the identification algorithm. The imprints simulated using the parameter values are also compared with the target shape at various stages during the identification .

Table 2: Identified Voce parameters using different initial points, $E=70 \mathrm{GPa}, \nu=0.33$ and $h_{\max }=0.1 \mathrm{~mm}$

\begin{tabular}{ccccccc}
\hline Identified material & \multirow{2}{*}{$\sigma_{y}(\mathrm{MPa})$} & $\frac{\left|\sigma_{y}^{*}-\sigma_{y}\right|}{\sigma_{y}^{*}}$ & \multirow{2}{*}{$Q(\mathrm{MPa})$} & $\frac{\left|Q^{*}-Q\right|}{Q^{*}}$ & & $\frac{\left|\gamma^{*}-\gamma\right|}{\gamma^{*}}$ \\
\hline $\mathrm{M}_{4}$ & 300.50 & $0.17 \%$ & 200.88 & $0.44 \%$ & 13.70 & $2.14 \%$ \\
$\mathrm{M}_{5}$ & 260.29 & $13.24 \%$ & 201.15 & $0.57 \%$ & 41.50 & $196.43 \%$ \\
\hline
\end{tabular}

Next, to rule out any possible influence of the choice of the initial point in inverse optimization, we also select $\boldsymbol{\mu}_{B}$ : $(200 \mathrm{MPa}, 600 \mathrm{MPa}, 140)$ for the algorithm, and the identified material is denoted by $\mathrm{M}_{5}$. The convergence histories are shown in Fig.9, and the final identified values are compared with those obtained when starting from $\boldsymbol{\mu}_{A}$, in Tab.2. Despite stabilizing around a particular set of values, only ONE of the three parameters $(Q)$ converges correctly to the reference value. A large deviation is seen for $\gamma$ in particular, with an error of up to $200 \%$ ! Nevertheless, Fig.10 shows a steady decline in the discrepancy between the identified and target imprints and we end up with an acceptable error of 0.002 .

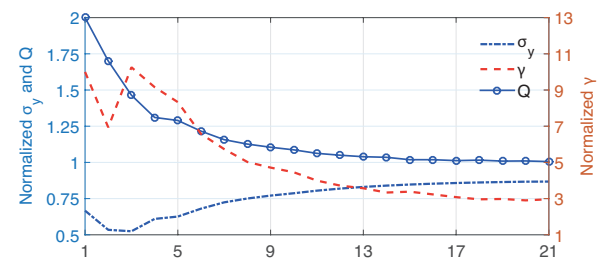

Fig. 9: Convergence summary for the three Voce parameters $\left(M_{5}\right)$ normalized by their corresponding reference values (initial point $\boldsymbol{\mu}_{B}$ ).

For the two identified materials, we reconstruct their constitutive behaviors using the identified parameters listed in Tab.2. We observe that, even in the case where both measurement and model errors are absent, it appears difficult to accurately/uniquely identify the differences in the post-yield properties of the Voce law using only the indentation imprint. In other words, the two identified parameter sets supposedly form a mystical pair, both minimizing the discrepancy with the "experimental" imprint. 


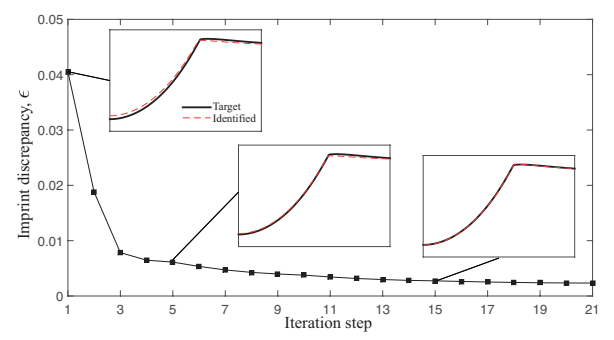

Fig. 10: Minimization of the discrepancy between the simulated $\left(\mathrm{M}_{5}\right)$ and target (pseudo-experimental) imprints (initial point $\boldsymbol{\mu}_{B}$ ).

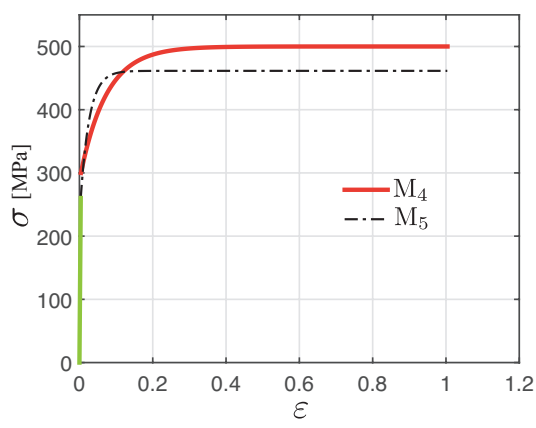

Fig. 11: A comparison of tensile curves for $\left(\mathrm{M}_{4}, \mathrm{M}_{5}\right)$ rebuilt using Voce law with the identified parameters.

The corresponding indentation $P$ - $h$ curves are now compared to check whether the two mystical sibling materials can be distinguished from each other by using all available indentation responses. We note that, despite the constitutive behaviors of the two materials being clearly distinct, the two indentation curves are nearly overlapping in Fig.12. In this regard, the combination of $P$ - $h$ curve and imprint [13] does not seem helpful in the current case. We must then pose the following questions:

Q1: is this failure in characterization due to the reduced-order space and/or the optimization algorithm used in the inverse analysis?

Q2: does the collected/measured information from the indentation response interpret material plasticity sufficiently?

We will address these two questions in the following section to investigate the source of this non-uniqueness problem for Voce hardening materials.

\section{Investigating the source of the non-uniqueness problem in identifying Voce parameters}

In this section, to first answer the question Q1 raised at the end of Sect.5, we verify the efficacy of the shape-manifold identification procedures for the three-parameter law using tensile curves in place of the indentation responses. 

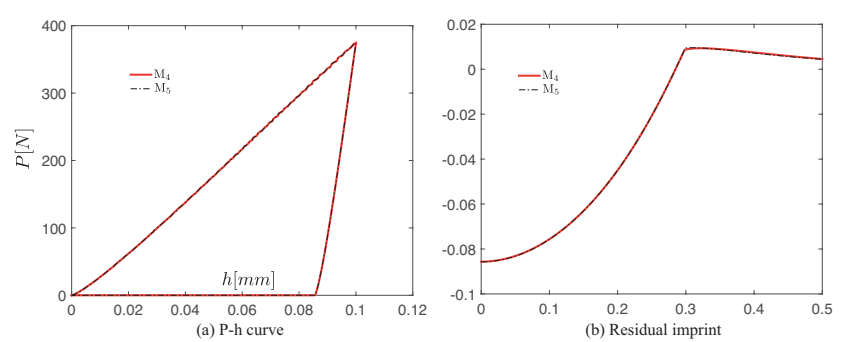

Fig. 12: A comparison of indentation responses for a mystical material pair $\left(\mathrm{M}_{4}, \mathrm{M}_{5}\right)$ for Voce law.

6.1 Verifying the efficacy of the identification procedure

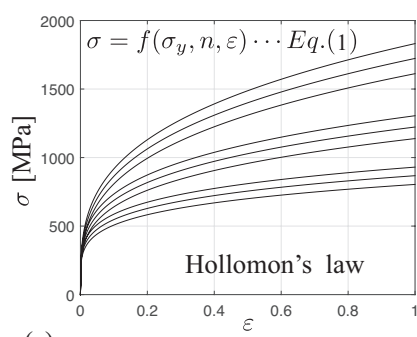

(a)

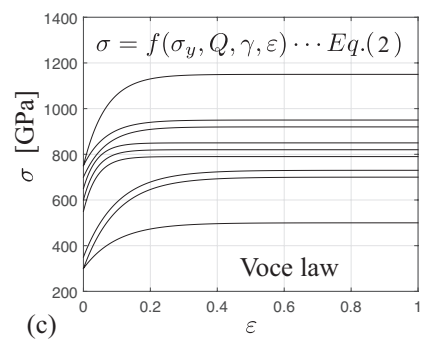

2D hyper-surface

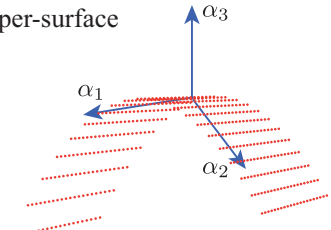

(b)

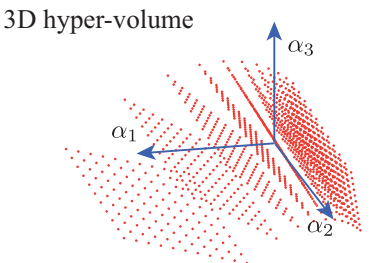

(d)

Fig. 13: Illustrative stress-strain curves and the corresponding low-dimensional embeddings for (a)-(b) Hollomon's power law hardening, and (c)-(d) Voce law hardening.

We temporarily leave the indentation context and revisit the uniaxial stress strain $(\sigma-\varepsilon)$ curves, directly considering them as high-dimensional MDS input "shapes". We show a series of representative stress-strain curves for the twoparameter Hollomon's (Fig.13 (a)) and the three-parameter Voce law (Fig.13 (c)), based on an 11 levels full factorial DoE within the following parameter intervals: $\sigma_{y} \in[200 \mathrm{MPa}, 240 \mathrm{MPa}]$ and $n \in[0.2,0.3]$ for Hollomon's law, and $\sigma_{y} \in[300 \mathrm{MPa}, 800 \mathrm{MPa}], Q \in[200 \mathrm{MPa}, 400 \mathrm{MPa}]$ and $\gamma \in[10,60]$ for Voce hardening. The resulting patterns of points in the embedded space allow us to conclude that: 
- for Hollomon's power law hardening materials, we observe that the projections are arranged in regular fashion, showing an intrinsic 2D hyper-surface, Fig.13(b);

- for Voce hardening law, we observe that all the material behavior curves are projected to a 3D cloud of points, taking the form of a multi-layer sandwich, Fig.13(d) (indicating an intrinsic dimensionality of 3 as expected).

In both cases, the parameters are thus considered independent and may be identified uniquely from the stress-strain curve using the interpolation technique.

\subsection{Identifying Voce parameters from $\sigma-\varepsilon$ curve}

For the sake of completeness, we carry out eight different identification procedures for the Voce parameters by directly correlating the shape of stressstrain curve using different starting points. The $\sigma-\varepsilon$ curve obtained from the set $\left(\sigma_{y}, Q, \gamma\right)=(300,200,25)$ is used as the "target shape", and the parameter set to be identified is located (by design) at the centroid of the cube enclosed by the eight initial points as vertices, as illustrated in Fig.14.

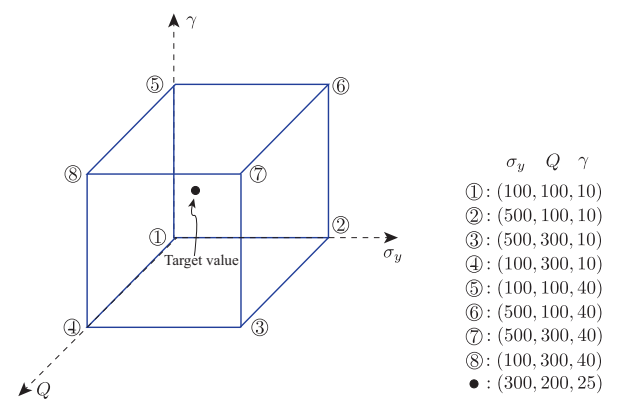

Fig. 14: The "target" material (black dot) and eight different parameter combinations chosen as initial points in parameter space.

Tab.3 lists the final estimates for the three parameters and the number of iteration steps for each corresponding case. We note that, in all eight cases, we always converge to a unique combination of parameters. A maximal difference of $0.16 \%$ and $0.1 \%$ is observed for $\gamma$ and $Q$ with respect to their respective target values. Analogously, other identification procedures have also been carried out considering Hollomon's power law and similar conclusions were reached. Fig.13 and the corresponding identification results tabularized in Tab.3 may promote us to correlate a relationship between the number of identifiable parameters and the intrinsic dimensionality of the manifold of admissible shapes within the reduced-order space.

On the other hand, it goes without saying that no iteration is really required for probing material parameters from the uniaxial stress-strain consti- 
Table 3: Voce parameters identified from various starting points using the stress-strain curve.

\begin{tabular}{ccccc}
\hline Initial points & $\sigma_{y}$ & $\mathrm{Q}$ & $\gamma$ & Iter No. \\
\hline (1) & 299.96 & $200.08 \%$ & 25.02 & 34 \\
(2) & 300.08 & $199.86 \%$ & 24.96 & 34 \\
(3) & 299.92 & $200.12 \%$ & 25.04 & 25 \\
(4) & 299.93 & $200.10 \%$ & 25.04 & 21 \\
(5) & 299.93 & $200.19 \%$ & 25.03 & 23 \\
(6) & 299.97 & $200.03 \%$ & 25.02 & 28 \\
(7) & 299.96 & $200.11 \%$ & 25.02 & 23 \\
(8) & 300.02 & $199.95 \%$ & 24.99 & 33 \\
\hline Target & 300 & 200 & 25 & - \\
\hline Max error & $0.03 \%$ & $0.10 \%$ & $0.16 \%$ & - \\
\hline
\end{tabular}

tutive law and that the identification can be readily accomplished by simple curve fitting provided that the target $\sigma-\varepsilon$ curve has already been obtained from a tensile test. That said, the identification procedures presented above, though redundant and bordering on overkill for this simple problem, still help us to assess the independence of constitutive parameters and to rule out any possibility of an inefficiency or other issues surrounding either the manifold hypothesis or identification algorithm we have used.

6.3 The inadequacy of "single depth" indentation for characterizing Voce material
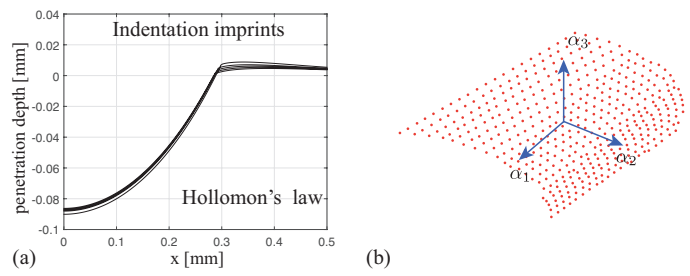

(b)
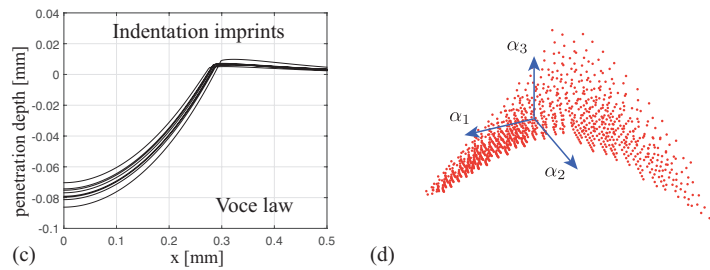

Fig. 15: High-dimensional indentation responses and the corresponding embeddings for different constitutive laws: (a)-(b) Hollomon's law, and (c)-(d) Voce law 
We return now to the indentation responses: residual imprints for Hollomon's and Voce law. An analogous procedure to the one explained in Sect.6.1 is followed. On the left of Fig.15, we show collections of representative indentation responses for varying the material parameters according to the DoE in Sect.6.1. The indentation responses projected into the reduced shape space are shown on the right. We derive the following observations:

- for residual imprints obtained according to Hollomon's power law, Fig.15(a), their 2D distribution in reduced shape-space confirms that the non-uniqueness issue does not present itself in this case, Fig.15(b);

- for imprints based on Voce law (that should be governed by three parameters), Fig.15(c), the point cloud appears to follow a more complex behavior, locally approaching a quasi-2D hyper-surface, Fig.15 (d)! As a consequence, the combination of material parameters identified assuming the Voce law is likely unreliable, confirming the results obtained in Sect.5.

By excluding the inefficacy of manifold protocol in identification, we conclude that the possibility of unique identification of Voce materials rests with the employed measurements, which may or may not interpret all material plasticity in one indentation test. We have by now answered the question Q2 raised in Section 5.

\subsection{The concept of "true" and "false" mystical pairs for Voce materials}

For the 1331 imprint snapshots in Fig. 15 (d), we plot (in the $\sigma_{y}-Q-\gamma$ space in Fig.16) the map of the goodness of fit, $g$, Eq.(10), w.r.t. the identification target $\mathrm{M}_{\mathrm{t}}\left(\sigma_{y}=550 \mathrm{MPa}, Q=300 \mathrm{MPa}\right.$ and $\left.\gamma=110\right)$. All materials that produce nearly identical imprints (with $g>0.995$ ) - the blue points in the figure - are located within a "husk of corn"-shaped zone, stretching along the diagonal direction (dashed-line). This is similar to the results obtained for the non-unique identification of Hollomon's power law parameters for conical indenters in $[15,5]$.

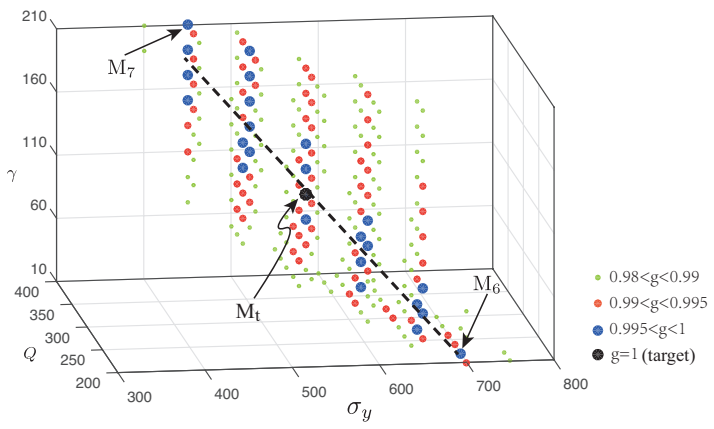

Fig. 16: Goodness of fit (Eq.10) for the 1331 Voce imprints using a spherical indenter. 
We choose two potential siblings of $\mathrm{M}_{\mathrm{t}}$, i.e., $\mathrm{M}_{6}$ and $\mathrm{M}_{7}$, with extreme values of $\gamma$. Their corresponding constitutive behaviors are compared in Fig.17. For $\mathrm{M}_{\mathrm{t}}$ and $\mathrm{M}_{7}$, the $\sigma-\varepsilon$ curves are very close to each other and their saturation stresses at high strain level are the same. We call them a "false mystical pair" due to the similarity of their constitutive behaviors. We suggest for this group of mystical materials that the difficulty in unique identification seems to be related to the parameterization of certain materials, rather than the indentation mechanism, partly due to the compensation of underestimating of $\sigma_{y}$ by overestimating $\gamma$. Nevertheless, a clear difference can be observed between that of $\mathrm{M}_{\mathrm{t}}$ and $\mathrm{M}_{6}$, and we term them as a "true mystical pair", referring to materials with completely different rheological behaviors, yet with nearly indistinguishable indentation responses.

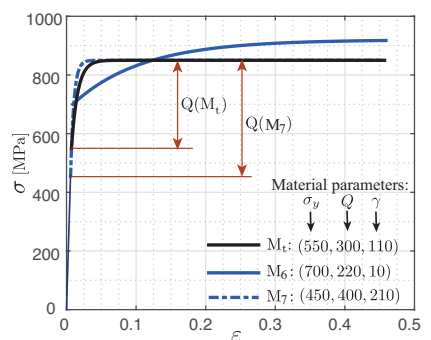

Fig. 17: Two mystical material pairs using the Voce hardening law: $\left(\mathrm{M}_{\mathrm{t}}, \mathrm{M}_{6}\right)$ the true mystical material pair, and $\left(\mathrm{M}_{\mathrm{t}}, \mathrm{M}_{7}\right)$ the false mystical material pair.

Besides, by examining the corresponding material parameters, we note that there appear to be more mystical siblings for materials with a higher $\gamma$. This is understandable, since $\gamma$ dominates mainly the transitional portion of the $\sigma-\varepsilon$ curve, and for materials with higher $\gamma$, this transitional phase is relatively short and thus is difficult to be properly captured by the indentation test.

\section{Alternative identification protocols for Voce law}

\subsection{Dual-sharp indentatation}

Inspired by $[14,16,11]$, dual-sharp indentation may be helpful to uniquely determine the parameters. As proposed in [51], for each indenter shape, a $g$-map characterizing the similarity of imprints indented for different materials is first generated in form of "cloud" of points within the design space. Then, after superimposing multiple clouds (corresponding to different indenter shapes), a "master cloud" is obtained (with the value associated with each point equal to the average/minimum for that point). Finally, the best fit solution would be the point in this master cloud with the highest value of $g$.

We therefore build two other databases for the two sharp conical indenters with apex angle $\theta=70.3^{\circ}$ and $\theta=63.14^{\circ}$, respectively. Contrary to what 

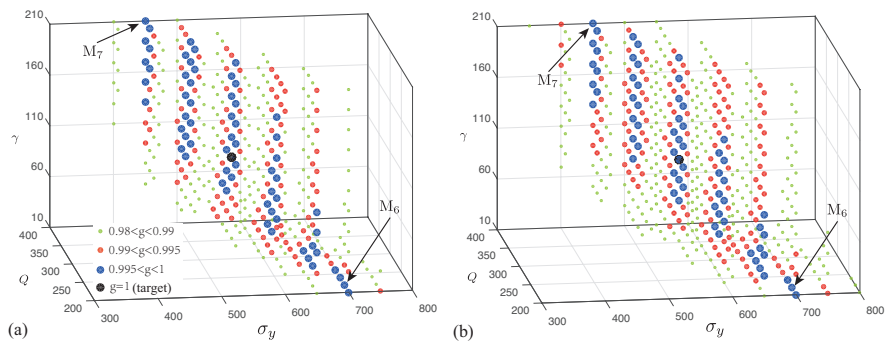

Fig. 18: Goodness of fit for Voce imprints using dual-sharp indentation with: (a) $\theta=70.3^{\circ}$, and (b) $\theta=63.14^{\circ}$.

one may expect, $M_{6}$ and $M_{7}$ remain mystical siblings for $M_{t}$, as indicated in Fig.18, and even worse, additional mystical material siblings are observed, with the distribution of these siblings appearing to follow the same direction as indicated in Fig.16. Even when the two point clouds are superimposed, the two materials are still present in the master cloud. This could indicate that multiindentation may not improve the identification results, and the unique inverse identification of Voce hardening parameters remains an unsolved problem to this day. Alternative indentation techniques must be sought to distinguish these mystical materials.

\subsection{Multi-depth indentation protocol}

Since in Fig.5 the difference between mystical materials is observed around the pile-up zone, we speculate by consequence this difference may be more pronounced with deeper indenter penetration, producing an alternative way to differentiate between mystical siblings of Voce hardening materials. Therefore, we propose in this subsection performing multiple indentation tests using different indentation depths.

Table 4: Comparison of pile-up heights for $\mathrm{M}_{4}$ and $\mathrm{M}_{5}$ with diverse penetration depths.

\begin{tabular}{cccc}
\hline Indentation depth & $h_{\max }=0.1 \mathrm{~mm}$ & $h_{\max }=0.15 \mathrm{~mm}$ & $h_{\max }=0.2 \mathrm{~mm}$ \\
\hline $\mathrm{M}_{4}$ & 0.0093 & 0.0169 & 0.0253 \\
$\mathrm{M}_{5}$ & 0.0096 & 0.0184 & 0.0285 \\
Difference & 0.0003 & 0.0015 & 0.0032 \\
\hline Relative difference & $3.13 \%$ & $8.15 \%$ & $11.23 \%$ \\
\hline
\end{tabular}

For the mystical material pair $\left(\mathrm{M}_{4}, \mathrm{M}_{5}\right)$ in Tab.2, we perform three indentation tests using the same spherical indenter. The maximal penetration depths are designed to equal to $0.1 \mathrm{~mm}, 0.15 \mathrm{~mm}$ and $0.2 \mathrm{~mm}$, respectively. The indentation responses are observed in Fig.19. Also, in Tab.4, we list the maximal pile-up heights observed from different imprints, and we note that 
$\mathrm{M}_{4}$ and $\mathrm{M}_{5}$ become distinguishable in view of their increasing difference of the pile-up heights.

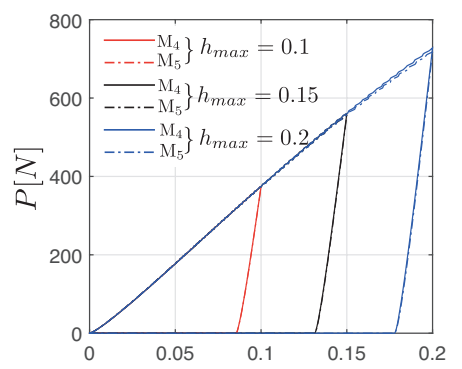

(a) P-h curve

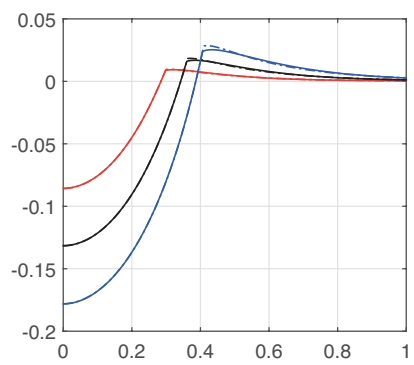

(b) Residual imprint

Fig. 19: Multi-depths indentation responses for the mystical material pair $\left(\mathrm{M}_{4}, \mathrm{M}_{5}\right)_{R=0.5}$ (Fig.12).

Then, following the same routine as for the dual-sharp indentation, we combine the three databases obtained for different penetration depths by superimposing the clouds of points in the map of goodness of fit (Fig.20) to obtain a master cloud. We note that more than one material parameter sets lead to almost indistinguishable imprints for all levels of loads, which means the mystical materials, either "true" or "false", are still present. However, less mystical siblings (i.e., the blue points) are observed compared with either single (Fig.16) or dual-sharp indention (Fig.18). More importantly, it is observed that $\mathrm{M}_{6}$, one of the true mystical material w.r.t. $\mathrm{M}_{\mathrm{t}}$, is excluded from the mystical siblings.

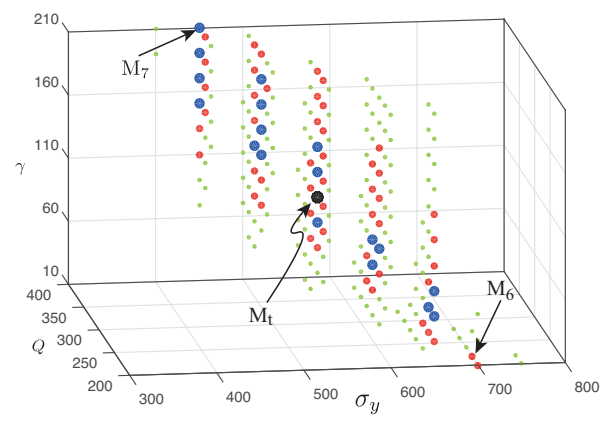

Fig. 20: Goodness of fit for indentation imprints using multi-depths indentation: the "true mystical material" $\mathrm{M}_{6}$ is readily separated from others

In Fig.21, the material behaviors of all those mystical siblings are then plotted in blue lines. We note that all of them are constructed with big values of $\gamma(\gamma \geq 50)$ while the saturation stresses are almost the same, belonging to 
the "false mystical materials" distinguished in Sect.6.3. On the other hand, we plot in the same figure the stress-strain curve of $\mathrm{M}_{6}$ (red dashed line), which as a result can be readily distinguished from the "false mystical materials". Thus, the "true mystical material" $\mathrm{M}_{6}$ is demystified with multi-depths indentation.

To conclude, we consider multi-depths indentation as a possible approach to make the difference between "true" and "false" mystical pairs for materials following Voce law. The presence of false mystical siblings partially takes roots in the parametrization of the constitutive law, and partially due to the inefficiency of indentation in capturing certain material properties, $\gamma$ for instance. However, this conclusion is based on global manifold observation and purely synthetic data, more verifications are still required.

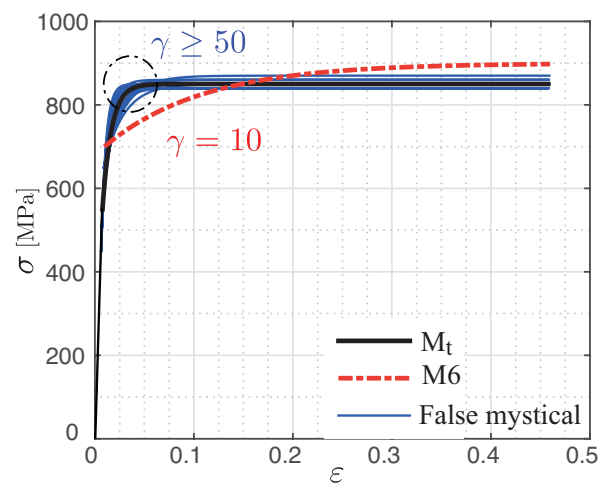

Fig. 21: "True mystical material" that is distinguished with multi-depth indentation.

\section{Conclusions and perspectives}

In this paper, we directly addressed the issue of non-uniqueness encountered in indentation-based material characterization, by analyzing the indentation responses (with particular focus on the imprint) in a reduced dimensional space. Two different material hardening laws, the two-parameter Hollomon's power law and the three-parameter Voce law, were considered. The salient features of this work may be summarized as follows:

- We have proposed a MDS-based protocol for analyzing indentation responses in a reduced-order shape-space, while preserving pairwise distances between indentation instances. We have successfully demystified the "mystical siblings" of Hollomon's law by using the residual imprint, even with a single self-similar indenter. This conclusion is in line with the unique identification of two plastic properties of C100 steel and AU4G aluminum alloy in [34].

- We distinguished between two types of mystical pairs for the Voce law: (a) the "false mystical pair" which may be explained by the similarity of the 
uniaxial stress-strain curves, and (b) the "true mystical pair", whose constitutive behaviors are completely different, yet the indentation responses virtually indistinguishable.

- Dual-sharp indentation, though useful for identifying Hollomon's power law parameters, may not improve the identification results for Voce hardening materials. However, multi-depth indentation probably allows us to differentiate between true and false mystical siblings.

Admittedly, these observations have been gleaned from an investigation using synthetic (simulated) data. Since noise will inevitably be introduced when using real experimental responses, further verifications using experimental indentation responses are required. Besides, the indentation test modeled in our current work is on a meso-scale level (penetration depth around $0.1 \mathrm{~mm}$ ), and the impact of crystal orientation, crystal size as well as work-hardened layer can be non-negligible and shall be carefully considered when the test is carried out down to nano-scale. As future work, we also recommend that the contact between the indenter and specimen be characterized simultaneously with the material properties, given the non-negligible effect of the friction coefficient on the indentation imprints.

Acknowledgements This study was funded by National Natural Science Foundation of China (Ref No. 11620101002) and the China scholarship Council (Ref No. 201404490062). This work has also been supported by the French National Research Agency (ANR) through the research grant Micromorfing, ANR-14-CE07-0035-01.

\section{Conflict of interest}

The authors declare that they have no conflict of interest.

\section{References}

1. D. Tabor, The hardness of metals, vol. 10 (ClarendonP, 1951)

2. A. Mukhopadhyay, Journal of Materials Science Letters 18(4), 333 (1999)

3. R.C. Paietta, S.E. Campbell, V.L. Ferguson, Journal of biomechanics 44(2), 285 (2011)

4. S. Kamali-Bernard, D. Keinde, F. Bernard, Key Engineering Materials 617, 14 (2014)

5. C. Moussa, O. Bartier, G. Mauvoisin, X. Hernot, J.M. Collin, G. Delattre, Surface and Coatings Technology 258(0), 782 (2014)

6. M. Sebastiani, A. Cusmà, E. Bemporad, F. Carassiti, Surface Engineering 30(1), 41 (2014)

7. X. Chen, J.J. Vlassak, Journal of Materials Research 16(10), 2974 (2001)

8. K. Tho, S. Swaddiwudhipong, Z. Liu, K. Zeng, J. Hua, Journal of Materials Research 19, $2498(2004)$

9. S. Swaddiwudhipong, K. Tho, Z. Liu, K. Zeng, International Journal of Solids and Structures 42(1), 69 (2005)

10. J. Alkorta, J. Martinez-Esnaola, J.G. Sevillano, Journal of materials research 20(02), $432(2005)$

11. X. Chen, N. Ogasawara, M. Zhao, N. Chiba, Journal of the Mechanics and Physics of Solids 55(8), 1618 (2007) 
12. J. Phadikar, T. Bogetti, A.M. Karlsson, International journal of solids and structures $\mathbf{5 0}(20), 3242(2013)$

13. L. Meng, P. Breitkopf, G. Le Quilliec, International Journal of Solids and Structures 106, $13(2017)$

14. Y.P. Cao, J. Lu, Acta Materialia 52(5), 1143 (2004)

15. C. Moussa, X. Hernot, O. Bartier, G. Delattre, G. Mauvoisin, Materials Science and Engineering: A 606, 409 (2014)

16. N. Chollacoop, M. Dao, S. Suresh, Acta materialia 51(13), 3713 (2003)

17. J. Dean, T. Clyne, Mechanics of Materials (2016)

18. Y.H. Lee, D. Kwon, Scripta Materialia 49(5), 459 (2003)

19. D. Carnelli, R. Lucchini, M. Ponzoni, R. Contro, P. Vena, Journal of biomechanics 44(10), 1852 (2011)

20. L. Shen, Y. He, D. Liu, Q. Gong, B. Zhang, J. Lei, Journal of Materials Research 30(08), $1078(2015)$

21. J.J. Schwiedrzik, P. Zysset, Journal of biomechanics 48(2), 210 (2015)

22. L. Meng, B. Raghavan, O. Bartier, X. Hernot, G. Mauvoisin, P. Breitkopf, Mechanics of Materials 107, 31 (2017)

23. M. Bocciarelli, G. Bolzon, Materials Science and Engineering: A 448(1), 303 (2007)

24. G. Bolzon, B. Molinas, M. Talassi, Strain 48(6), 517 (2012)

25. D. González, E. Cueto, F. Chinesta, Annals of biomedical engineering pp. 1-11 (2015)

26. Y. Hou, T. Sapanathan, A. Dumon, P. Culière, M. Rachik, Computational Materials Science 123, 188 (2016)

27. B. Raghavan, L. Xia, P. Breitkopf, A. Rassineux, P. Villon, Computer Methods in Applied Mechanics and Engineering 265, 174 (2013)

28. L. Xia, B. Raghavan, P. Breitkopf, W. Zhang, Applied Mathematics and Computation 224, $450(2013)$

29. B. Raghavan, G. Le Quilliec, P. Breitkopf, A. Rassineux, J.M. Roelandt, P. Villon, International Journal of Material Forming 7(4), 487 (2014)

30. Y. Hou, T. Sapanathan, A. Dumon, P. Culière, M. Rachik, Engineering Fracture Mechanics 188, 232 (2018)

31. Y. Tie, Y. Hou, C. Li, X. Zhou, T. Sapanathan, M. Rachik, Composite Structures 190, $179(2018)$

32. C. Zhang, B. Wang, Journal of Materials Research 27(20), 2624 (2012)

33. G. Le Quilliec, B. Raghavan, P. Breitkopf, Computer Methods in Applied Mechanics and Engineering 285, 621 (2015)

34. L. Meng, P. Breitkopf, B. Raghavan, G. Mauvoisin, O. Bartier, X. Hernot, Computer Methods in Applied Mechanics and Engineering 297, 239 (2015)

35. L. Meng, P. Breitkopf, G. Le Quilliec, B. Raghavan, P. Villon, Archives of Computational Methods in Engineering pp. 1-21 (2016)

36. Y.T. Cheng, C.M. Cheng, Journal of Materials Research 14(09), 3493 (1999)

37. C. Moussa, X. Hernot, O. Bartier, G. Delattre, G. Mauvoisin, Journal of Materials Science 49(2), 592 (2014)

38. K.D. Bouzakis, N. Michailidis, S. Hadjiyiannis, G. Skordaris, G. Erkens, Materials characterization 49(2), 149 (2002)

39. K. Tunvisut, E. Busso, N. O'dowd, H. Brantner, Philosophical Magazine A 82(10), 2013 (2002)

40. J. Bressan, A. Tramontin, C. Rosa, Wear 258(1), 115 (2005)

41. Y.T. Cheng, C.M. Cheng, Materials Science and Engineering: R: Reports 44(4), 91 (2004)

42. H. Jin, D. Lloyd, Metallurgical and Materials Transactions A 35(13), 997 (2004)

43. C.G. Shastry, M. Mathew, K.B.S. Rao, S. Mannan, International Journal of Pressure Vessels and Piping 81(3), 297 (2004). DOI http://dx.doi.org/10.1016/j.ijpvp.2003.11. 016

44. A. Nayebi, O. Bartier, G. Mauvoisin, R.E. Abdi, International Journal of Mechanical Sciences 43(11), 2679 (2001)

45. A. Nayebi, R.E. Abdi, O. Bartier, G. Mauvoisin, Mechanics of Materials 34(4), 243 (2002)

46. F. Pöhl, S. Huth, W. Theisen, Journal of the Mechanics and Physics of Solids 66, 32 (2014) 
47. M. Mata, J. Alcala, Journal of the Mechanics and Physics of Solids 52(1), 145 (2004)

48. G. Bolzon, G. Maier, M. Panico, International Journal of Solids and Structures 41(11), 2957 (2004)

49. F. Salmoiraghi, F. Ballarin, G. Corsi, A. Mola, M. Tezzele, G. Rozza, (ECCOMAS, 2016)

50. M. Wang, J. Wu, Y. Hui, Z. Zhang, X. Zhan, R. Guo, Materials Science and Engineering: A 679, 143 (2017)

51. J. Dean, T. Clyne, Mechanics of Materials 105, 112 (2017) 